\title{
The impact of the Ambulatory Care Unit (ACU) on 'true admission avoidance' at Basingstoke and North Hampshire Hospital
}

\author{
Authors: Sebastien Ellis, ${ }^{A}$ Carl Heffernan ${ }^{B}$ and Channa Nadarajah ${ }^{C}$
}

\begin{abstract}
Aims
The Ambulatory Care Unit (ACU) is a recent addition to Basingstoke and North Hampshire Hospital. In January 2017, additional acute medical consultants were appointed to help develop the service. The clinic is consultant led and operates Monday-Friday from 09.00-18.00. ACU accepts referrals directly from GPs and operates a push/pull system for the Emergency Department (ED). Out-of-hours referrals can be made via the medical registrar on call. Before expanding the service further, we studied the true impact of ACU by looking not only at patients diverted from the medical take, but also at 30-day readmission rates and mortality, thereby reflecting 'true' admission avoidance (TAA).
\end{abstract}

\section{Methods}

Data were collected prospectively from January to June 2017. We measured the number of patients diverted from the medical take to $A C U$ and the number of patients who subsequently required admission. Patients were followed up at 30 days to measure readmission rates and mortality. TAA was calculated using the following formula:

\author{
TAA $(\%)=(($ new ACU patients + follow-up ACU patients $)$ \\ - (same-day ACU admissions + 30-day ACU \\ readmissions) $\div$ total medical referrals) $\times 100$
}

Results

Table 1 shows the monthly breakdown of clinical activity in ACU. Over 6 months, $20.3 \%$ of patients were diverted from the medical take to ACU. Of those seen via ACU, $10.7 \%$ required hospital admission. The 30 -day readmission rate was $2.0 \%$ and TAA was $24.7 \%$ (Table 1).

\section{Conclusions}

ACU diverts a significant proportion of the medical take. To study the true impact of ACU 30-day readmission rates, TAA and mortality should also be measured. Our data suggest that ACU can deliver significant TAA while maintaining low readmission rates and mortality. Our service is due to expand further in March 2018 and these data provide supporting evidence in terms of both clinical effectiveness and patient safety.

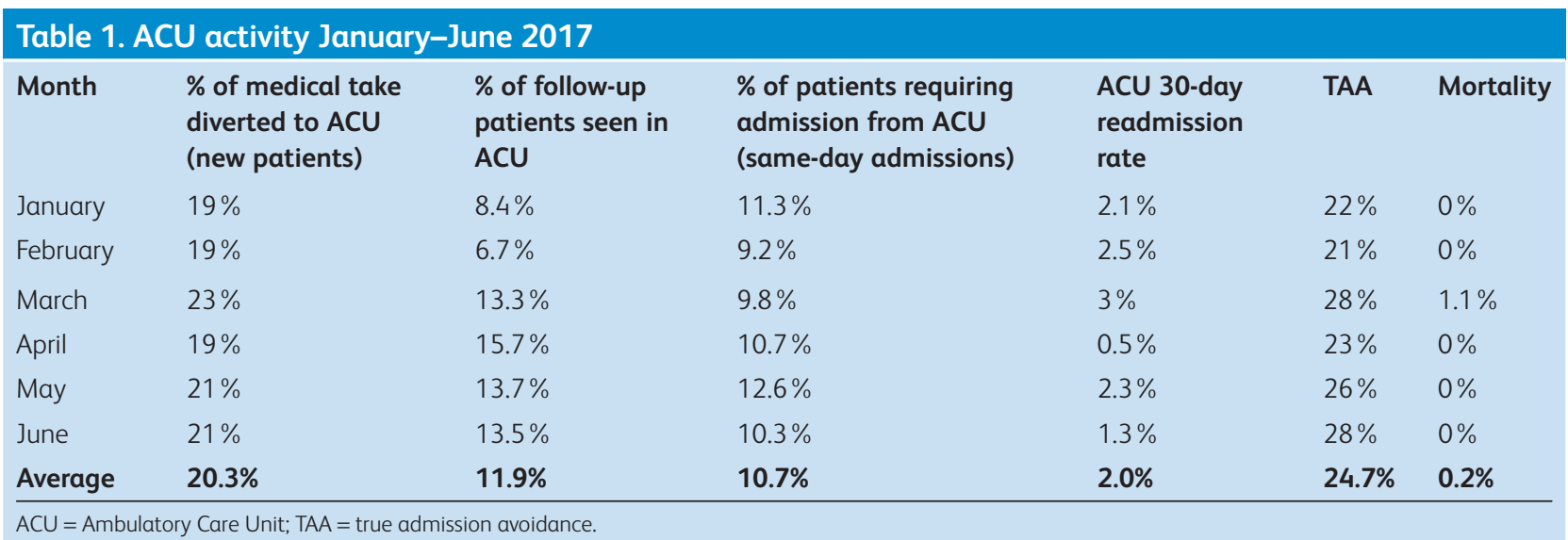

Authors: ${ }^{A}$ University Hospital Southampton NHS Foundation Trust,

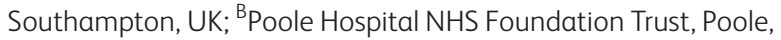
UK; ' ${ }^{C}$ Hampshire Hospitals NHS Foundation Trust, Basingstoke, UK

\section{Conflict of interest statement}

None declared. 